\title{
Quasi-Grammian Solutions of the Generalized Coupled Dispersionless Integrable System
}

\author{
Bushra HAIDER and Mahmood-ul HASSAN \\ Department of Physics, University of the Punjab, \\ Quaid-e-Azam Campus, Lahore-54590, Pakistan \\ E-mail: bushrahaider@hotmail.com,mhassan.physics@pu.edu.pk \\ URL: http://pu.edu.pk/faculty/description/526/, \\ http://www.pu.edu.pk/faculty/description/538/
}

Received June 22, 2012, in final form October 10, 2012; Published online November 08, 2012 http://dx.doi.org/10.3842/SIGMA.2012.084

\begin{abstract}
The standard binary Darboux transformation is investigated and is used to obtain quasi-Grammian multisoliton solutions of the generalized coupled dispersionless integrable system.
\end{abstract}

Key words: integrable systems; binary Darboux transformation; quasideterminants

2010 Mathematics Subject Classification: 70H06; 22E99

\section{Introduction}

The interest in dispersionless integrable systems is due to their wide range of applicability in various fields of mathematics and physics $[1,2,8,16,17,19,20,21,22,23,24,25,26,27,47$, 48, 50]. Most of the dispersionless integrable systems belong to a family where these systems arise as quasi-classical limit of ordinary integrable systems with a dispersion term $[1,2,8,22$, $23,25,27,47,48,50]$. But there are important examples of dispersionless integrable system which are referred to as dispersionless not in the sense mentioned above but due to the absence of dispersion term. The coupled dispersionless integrable systems and its generalizations are examples of such integrable systems $[16,17,19,20,21,24,26]$. The Darboux transformation of the generalized coupled dispersionless integrable system has been studied in a recent work [16].

The purpose of this paper is to study the standard binary Darboux transformation of the generalized coupled dispersionless integrable system and to derive exact solutions in terms of quasi-Grammians. We employ the method introduced in [15], construct standard binary Darboux transformation by introducing Darboux matrices of the system for the direct and the adjoint Lax pairs and then obtain binary Darboux matrix by composing the two Darboux transformations. We obtain the quasi-Grammian multisoliton solutions using the iterated binary Darboux transformations. We also consider the system based on Lie group $S U(N)$ and obtain explicit solutions of the system based on $S U(2)$.

The action of the generalized coupled dispersionless integrable system based on some nonAbelian Lie group $\mathcal{G}$ is given by

$$
I=\int d t d x \mathcal{L}\left(S, S_{x}, S_{t}\right)
$$

where the Lagrangian density $\mathcal{L}\left(S, S_{x}, S_{t}\right)$ is defined by

$$
\mathcal{L}=\operatorname{Tr}\left(\frac{1}{2} S_{x} S_{t}-\frac{1}{3} G\left[S,\left[S_{x}, S\right]\right]\right)
$$


where $S$ is a matrix field and $G$ is a constant matrix taking values in the non-Abelian Lie algebra $\mathfrak{g}$ of the Lie group $\mathcal{G}$. The matrix fields $S$ and $G$ are Lie algebra $\mathfrak{g}$ valued, i.e., $S=\phi^{a} T^{a}$ and $G=\kappa^{a} T^{a}$, where anti-hermitian generators $\left\{T^{a}, a=1,2, \ldots, \operatorname{dim} \mathfrak{g}\right\}$ of the Lie algebra $\mathfrak{g}$ obey $\left[T^{a}, T^{b}\right]=f^{a b c} T^{c}$ and $\operatorname{Tr}\left(T^{a} T^{b}\right)=-\delta^{a b}$. For any $X \in \mathfrak{g}, X=X^{a} T^{a}$. Note that $\phi^{a}=$ $\phi^{a}(x, t)$ is a vector field with components $\left\{\phi^{q}, a=1,2, \ldots, \operatorname{dim} \mathfrak{g}\right\}$ and $\kappa$ is the constant vector having components $\left\{\kappa^{a}, a=1,2, \ldots, \operatorname{dim} \mathfrak{g}\right\}$. The equation of motion of the generalized coupled dispersionless system as obtained from (1.1) is

$$
S_{x t}-\left[[S, G], S_{x}\right]=0 .
$$

For $\mathcal{G}=S U(2)$ we get from $(1.2)$

$$
q_{x t}+(r \bar{r})_{x}=0, \quad r_{x t}-2 q_{x} r=0, \quad \bar{r}_{x t}-2 q_{x} \bar{r}=0,
$$

where $q$ is a real valued function and $r$ is a complex valued function of $x$ and $t$. Here $\bar{r}$ denotes complex conjugate of $r$.

The generalized coupled dispersionless system (1.2) can be written as the compatibility condition of the following Lax pair

$$
\partial_{x} \psi=U(x, t, \lambda) \psi, \quad \partial_{t} \psi=V(x, t, \lambda) \psi,
$$

where $\psi \in \mathcal{G}$ and $\lambda$ is a real (or complex) parameter. The fields $U$ and $V$ are $n \times n$ matrix fields and are given by

$$
U(x, t, \lambda)=\lambda \partial_{x} S, \quad V(x, t, \lambda)=[S, G]+\lambda^{-1} G .
$$

The compatibility condition of the linear system (1.4) is the zero curvature condition

$$
\partial_{t} U(x, t, \lambda)-\partial_{x} V(x, t, \lambda)+[U(x, t, \lambda), V(x, t, \lambda)]=0 .
$$

Note that the above equation (1.5) is equivalent to the equation of motion (1.2). The Darboux transformation of the generalized coupled dispersionless system has been discussed in [16]. In the next section we will retrace the steps for the Darboux transformation for direct and adjoint spaces and then we will combine the two elementary Darboux transformations to obtain the standard binary Darboux transformation of the generalized coupled dispersionless system.

\section{Darboux transformation on the direct and adjoint Lax pairs}

In this section we discuss the Darboux transformation on the solutions to the direct and adjoint Lax pairs. For details of Darboux transformation see e.g. [3, 4, 5, 6, 7, 14, 18, 29, 30, 31, 32, 34, 35, 36, 37, 38, 39, 44, 46, 49]. The one-fold Darboux transformation on the matrix solution to the Lax pair (1.4) is defined by

$$
\tilde{\psi}(\lambda)=D\left(x^{+}, x^{-}, \lambda\right) \psi(\lambda),
$$

where $D(x, t, \lambda)$ is the Darboux matrix. We use the following ansatz for the Darboux matrix $D(x, t, \lambda)$

$$
D\left(x^{+}, x^{-}, \lambda\right)=\lambda^{-1} I-M\left(x^{+}, x^{-}\right),
$$

and $M\left(x^{+}, x^{-}\right)$is some $n \times n$ matrix field to be determined and $I$ is an $n \times n$ identity matrix. The Darboux matrix transforms the matrix solution $\psi$ in space $\mathcal{V}$ to a new solution $\tilde{\psi}$ in $\tilde{\mathcal{V}}$, i.e.

$$
D(\lambda): \mathcal{V} \rightarrow \tilde{\mathcal{V}}
$$


The new solution $\tilde{\psi}$ satisfies the Darboux transformed Lax pair

$$
\partial_{x} \tilde{\psi}=\tilde{U}(x, t, \lambda) \tilde{\psi}, \quad \partial_{t} \tilde{\psi}=\tilde{V}(x, t, \lambda) \tilde{\psi},
$$

where the matrix-valued fields $\tilde{U}$ and $\tilde{V}$ are given as

$$
\tilde{U}(x, t, \lambda)=\lambda \partial_{x} \tilde{S}, \quad \tilde{V}(x, t, \lambda)=[\tilde{S}, \tilde{G}]+\lambda^{-1} \tilde{G},
$$

and $\tilde{S}$ and $\tilde{G}$ are the Lie algebra valued transformed matrix fields. The covariance of the Lax pair (1.4) under Darboux transformation can be checked by substituting equation (2.1) in equations (2.4). The covariance implies the following Darboux transformation on the matrixvalued fields $S$ and $G$

$$
\begin{aligned}
& \tilde{S}=S-M, \\
& \tilde{G}=G .
\end{aligned}
$$

As mentioned earlier equation (2.6) shows that $G$ is a constant matrix and the matrix $M$ is subjected to satisfy the following equations

$$
\partial_{x} M M=\left[\partial_{x} S, M\right], \quad \partial_{t} M=[[S, G], M]+[G, M] M .
$$

The matrix $M$ can be written in terms of the solutions of the linear system [16]

$$
M=\Theta \Lambda^{-1} \Theta^{-1}
$$

where $\Theta$ is the particular matrix solution of the Lax pair defined by

$$
\Theta=\left(\psi\left(\lambda_{1}\right)|1\rangle, \ldots, \psi\left(\lambda_{n}\right)|n\rangle\right)=\left(\left|\theta_{1}\right\rangle, \ldots,\left|\theta_{n}\right\rangle\right),
$$

Each column $\left|\theta_{i}\right\rangle=\psi\left(\lambda_{i}\right)|i\rangle$ in $\Theta$ is a column solution of the Lax pair (1.4) when $\lambda=\lambda_{i}$, i.e., it satisfies

$$
\partial_{x}\left|\theta_{i}\right\rangle=\lambda_{i} \partial_{x} S\left|\theta_{i}\right\rangle, \quad \partial_{t}\left|\theta_{i}\right\rangle=[S, G]\left|\theta_{i}\right\rangle+\lambda_{i}^{-1} G\left|\theta_{i}\right\rangle,
$$

and $i=1,2, \ldots, n$. Assuming $\Lambda=\operatorname{diag}\left(\lambda_{1}, \ldots, \lambda_{n}\right)$, the equations $(2.8)$ can be written in matrix form as

$$
\partial_{x} \Theta=\partial_{x} S \Theta \Lambda, \quad \partial_{t} \Theta=[S, G] \Theta+G \Theta \Lambda^{-1} .
$$

The Darboux transformation of the generalized coupled dispersionless integrable system in terms of particular matrix solution $\Theta$ with the particular eigenvalue matrix $\Lambda$ is given as

$$
\tilde{\psi}=\left(\lambda^{-1} I-\Theta \Lambda^{-1} \Theta^{-1}\right) \psi, \quad \tilde{S}=S-\Theta \Lambda^{-1} \Theta^{-1}, \quad \tilde{G}=G .
$$

In terms of quasideterminants we can write the above expressions as

$$
\tilde{\psi}=\left|\begin{array}{cc}
\Theta & \psi \\
\Theta \Lambda^{-1} & \lambda^{-1} \psi
\end{array}\right|, \quad \tilde{S}=S+\left|\begin{array}{cc}
\Theta & I \\
\Theta \Lambda^{-1} & O
\end{array}\right|,
$$

where $O$ is an $n \times n$ null matrix. The result can be generalized to obtain $K$-fold Darboux transformation on matrix solution $\psi$ and can be written in terms of quasideterminant $\operatorname{as}^{1}$ (for

\footnotetext{
${ }^{1}$ The quasideterminant for an $N \times N$ matrix over a ring $R$ is defined as
}

$$
|X|_{i j}=\left|\begin{array}{cc}
X^{i j} & c_{j}^{i} \\
r_{i}^{j} & x_{i j}
\end{array}\right|=x_{i j}-r_{i}^{j}\left(X^{i j}\right)^{-1} c_{j}^{i},
$$

where for $1 \leq i, j \leq N, r_{i}^{j}$ is the row matrix obtained by removing $j$ th entry of $X$ from the $i$ th row. Similarly, $c_{j}^{i}$ is the column matrix containing $j$ th column of $X$ without $i$ th entry. There exist $N^{2}$ quasideterminants denoted by $|X|_{i j}$ for $i, j=1, \ldots, N$. For various properties and applications of quasideterminants in the theory of integrable systems, see e.g. $[9,10,11,12,28]$. 
more details see [16])

$$
\psi[K+1]=\psi[K]-\Theta[K] \Lambda_{K}^{-1} \Theta[K]^{-1} \psi[K]=\left|\begin{array}{cccc}
\Theta_{1} & \cdots & \Theta_{K} & \psi \\
\Theta_{1} \Lambda_{1}^{-1} & \cdots & \Theta_{K} \Lambda_{K}^{-1} & \lambda^{-1} \psi \\
\vdots & \ddots & \vdots & \vdots \\
\Theta_{1} \Lambda_{1}^{-K} & \cdots & \Theta_{K} \Lambda_{K}^{-K} & \lambda^{-K} \psi
\end{array}\right| .
$$

The expression for $S[K+1]$ is given as

$$
S[K+1]=S-\sum_{l=1}^{K} \Theta[K] \Lambda_{K}^{-1} \Theta[K]^{-1}=S+\left|\begin{array}{cccc}
\Theta_{1} & \cdots & \Theta_{K} & O \\
\vdots & \ddots & \vdots & \vdots \\
\Theta_{1} \Lambda_{1}^{-(K-2)} & \cdots & \Theta_{K} \Lambda_{K}^{-(K-2)} & O \\
\Theta_{1} \Lambda_{1}^{-(K-1)} & \cdots & \Theta_{K} \Lambda_{K}^{-(K-1)} & I \\
\Theta_{1} \Lambda_{1}^{-K} & \cdots & \Theta_{K} \Lambda_{K}^{-K} & O
\end{array}\right| .
$$

The $K$-fold Darboux transformation on the matrix solution $\psi$ can also be expressed in terms of Hermitian projectors $P[K]$, i.e.

$$
\psi[K+1]=\prod_{k=0}^{K}\left(I-\frac{\mu_{K-k+1}-\bar{\mu}_{K-k+1}}{\lambda^{-1}-\bar{\mu}_{K-k+1}} P[K-k+1]\right) \psi,
$$

where the Hermitian projection in this case is

$$
P[k]=\sum_{i=1}^{n} \frac{\left|\theta_{i}[k]\right\rangle\left\langle\theta_{i}[k]\right|}{\left\langle\theta_{i}[k] \mid \theta_{i}[k]\right\rangle}, \quad k=1,2, \ldots, K,
$$

with $P^{\dagger}[K]=P[K]$ and $P^{2}[K]=P[K]$.

Now we define the adjoint Darboux transformation. The equation of motion (1.2) and zero curvature condition (1.5) can also be written as compatibility condition of the following linear system (the adjoint Lax pair)

$$
\partial_{x} \phi=-\xi \partial_{x} S^{\dagger} \phi, \quad \partial_{t} \phi=-\left[S^{\dagger}, G^{\dagger}\right] \phi-\xi^{-1} G^{\dagger} \phi,
$$

which is obtained by taking the formal adjoint of the system (1.4). Note that in equation (2.10) $\xi$ is a real (or complex) parameter and $\phi$ is an invertible $n \times n$ matrix in the space $\mathcal{V}^{\dagger}=\{\phi\}$. The Darboux matrix $D(\xi)$ transforms the matrix solution $\phi$ in space $\tilde{\mathcal{V}}^{\dagger}$ to a new matrix solution $\tilde{\phi}$ in $\tilde{\mathcal{V}}^{\dagger}$, i.e.

$$
D(\xi): \mathcal{V}^{\dagger} \rightarrow \tilde{\mathcal{V}}^{\dagger}
$$

The one-fold Darboux transformation on the matrix solution $\phi$ is defined as

$$
\tilde{\phi} \equiv D(\xi) \phi=-\left(\xi^{-1} I-\Omega \Xi \Omega^{-1}\right) \phi,
$$

where $\Xi=\operatorname{diag}\left(\xi_{1}, \ldots, \xi_{n}\right)$ is the eigenvalue matrix. The matrix function $\Omega$ is an invertible non-degenerate $n \times n$ matrix and is given by

$$
\Omega=\left(\phi\left(\xi_{1}\right)|1\rangle, \ldots, \phi\left(\xi_{n}\right)|n\rangle\right)=\left(\left|\rho_{1}\right\rangle, \ldots,\left|\rho_{n}\right\rangle\right) .
$$

The $K$-fold Darboux transformation on matrix solutions $\phi, S^{\dagger}$ and $G^{\dagger}$ can be expressed as

$$
\phi[K+1]=\left|\begin{array}{cccc}
\Omega_{1} & \cdots & \Omega_{K} & \phi \\
\Omega_{1} \Xi_{1}^{-1} & \cdots & \Omega_{K} \Xi_{K}^{-1} & \xi^{-1} \phi \\
\vdots & \ddots & \vdots & \vdots \\
\Omega_{1} \Xi_{1}^{-K} & \cdots & \Omega_{K} \Xi_{K}^{-K} & \xi^{-K} \phi
\end{array}\right|,
$$




$$
S^{\dagger}[K+1]=S^{\dagger}+\left|\begin{array}{cccc}
\Omega_{1} & \cdots & \Omega_{K} & O \\
\vdots & \ddots & \vdots & \vdots \\
\Omega_{1} \Xi_{1}^{-(K-2)} & \ldots & \Omega_{K} \Xi_{K}^{-(K-2)} & O \\
\Omega_{1} \Xi_{1}^{-(K-1)} & \ldots & \Omega_{K} \Xi_{K}^{-(K-1)} & I \\
\Omega_{1} \Xi_{1}^{-K} & \cdots & \Omega_{K} \Xi_{K}^{-K} & O
\end{array}\right|, \quad G^{\dagger}[K+1]=G .
$$

In terms of the Hermitian projector we write the above expression as

$$
\phi[K+1]=\prod_{k=0}^{K}\left(I-\frac{\nu_{K-k+1}-\bar{\nu}_{K-k+1}}{\xi^{-1}-\bar{\nu}_{K-k+1}} P[K-k+1]\right) \phi,
$$

and the Hermitian projector in this case is defined as

$$
P[k]=\sum_{i=1}^{n} \frac{\left|\rho_{i}[k]\right\rangle\left\langle\rho_{i}[k]\right|}{\left\langle\rho_{i}[k] \mid \rho_{i}[k]\right\rangle}, \quad k=1,2, \ldots, K .
$$

By making use of equations (1.4) and (2.10) for the column solutions $\left|\theta_{i}\right\rangle$ and the row solutions $\left\langle\rho_{i}\right|$ of the direct and adjoint Lax pair respectively, it can be easily shown that the expressions (2.9) and (2.12) are equivalent.

\section{Standard binary Darboux transformation}

To define the binary transformation we follow the approach of $[13,40,41,42,43,45]$ and consider a space $\hat{\mathcal{V}}$, which is a copy of the direct space $\mathcal{V}$ and the corresponding solutions are $\hat{\psi} \in \hat{\mathcal{V}}$. Since it is a copy of the direct space, therefore the linear system, equation of motion and the zero curvature condition will have the similar form as given for the direct space. The equation of motion (1.2) and zero curvature condition (1.5) can also be written as the compatibility condition of the following linear system for the matrix solution $\hat{\psi}$

$$
\partial_{x} \hat{\psi}=\hat{U}(x, t, \lambda) \hat{\psi}, \quad \partial_{t} \hat{\psi}=\hat{V}(x, t, \lambda) \hat{\psi},
$$

where

$$
\hat{U}(x, t, \lambda)=\lambda \partial_{x} \hat{S}, \quad \hat{V}(x, t, \lambda)=[\hat{S}, \hat{G}]+\lambda^{-1} \hat{G} .
$$

We have taken the specific solutions $\Theta, \Omega$ for the direct and adjoint spaces $\mathcal{V}$ and $\mathcal{V}^{\dagger}$ respectively. The corresponding solutions for $\hat{\mathcal{V}}$ are $\hat{\Theta} \in \hat{\mathcal{V}}$ and $\hat{\phi} \in \hat{\mathcal{V}}^{\dagger}$. Also assuming that $i(\hat{\Theta}) \in \tilde{\mathcal{V}}^{\dagger}$, then from equations (2.3) and (2.11), we write the transformation as

$$
D^{(-1) \dagger}(\lambda): \mathcal{V}^{\dagger} \longrightarrow \tilde{\mathcal{V}}^{\dagger}
$$

Since $\phi \in \mathcal{V}^{\dagger}$, we have

$$
i(\hat{\Theta})=D^{(-1) \dagger}(\lambda) \phi .
$$

Also from $D^{\dagger}(\lambda)(i(\Theta))=0$, we obtain $i(\Theta)=\Theta^{(-1) \dagger}$ and similarly $i(\hat{\Theta})=\hat{\Theta}^{(-1) \dagger}$. Therefore we get from above equation

$$
\hat{\Theta}^{(-1) \dagger}=D^{(-1) \dagger}(\lambda) \phi, \quad \hat{\Theta}=\left(D^{(-1) \dagger}(\lambda) \phi\right)^{(-1) \dagger} .
$$

By using (2.2) and (2.7) in above equation

$$
\hat{\Theta}=\left(\left(\lambda^{-1} I-\Theta \Lambda^{-1} \Theta^{-1}\right)^{(-1) \dagger} \phi\right)^{(-1) \dagger}=\left(\lambda^{-1} I-\Theta \Lambda^{-1} \Theta^{-1}\right) \phi^{(-1)}
$$




$$
=\Theta\left(\lambda^{-1} I-\Lambda^{-1}\right) \Theta^{-1} \phi^{(-1) \dagger}=\Theta\left(\lambda^{-1} I-\Lambda^{-1}\right)\left(\phi^{\dagger} \Theta\right)^{-1}=\Theta \Delta^{-1},
$$

where the potential $\Delta$ is defined as

$$
\Delta(\psi, \phi)=\left(\phi^{\dagger} \Theta\right)\left(\lambda^{-1} I-\Lambda^{-1}\right)^{-1} .
$$

Similarly for adjoint space

$$
\hat{\Omega}=\Omega \Delta^{(-1) \dagger}
$$

we obtain

$$
\Delta(\psi, \Omega)=-\left(\lambda^{-1} I-\Xi^{(-1) \dagger}\right)^{-1}\left(\Omega^{\dagger} \psi\right) .
$$

By writing equations (3.3) and (3.4) in matrix form for the solutions $\Theta$ and $\Omega$, we get the following condition on $\Delta$

$$
\Xi^{(-1) \dagger} \Delta(\Theta, \Omega)-\Delta(\Theta, \Omega) \Lambda^{-1}=\Omega^{\dagger} \Theta
$$

where $\Delta$ is a matrix. An entry $\Delta_{i j}$ from equations (3.3), (3.4) and (3.5) is given as

$$
\Delta(\Theta, \Omega)_{i j}=\frac{\left(\Omega^{\dagger} \Theta\right)_{i j}}{\bar{\xi}_{i}^{-1}-\lambda_{j}^{-1}} .
$$

Now we define the Darboux matrix in hat space as

$$
\hat{D}(\lambda) \equiv\left(\lambda^{-1} I-\hat{S}\right)=\left(\lambda^{-1} I-\hat{\Theta} \Xi^{(-1) \dagger} \hat{\Theta}^{-1}\right),
$$

where

$$
\hat{D}(\lambda) \hat{\psi}=\tilde{\psi}
$$

We may summarize the above formulation as

$$
\begin{aligned}
& D(\lambda): \mathcal{V} \longrightarrow \tilde{\mathcal{V}}, \\
& \hat{D}(\lambda): \hat{\mathcal{V}} \longrightarrow \tilde{\mathcal{V}}, \\
& D(\xi): \mathcal{V}^{\dagger} \longrightarrow \tilde{\mathcal{V}}^{\dagger} .
\end{aligned}
$$

The effect of $\hat{D}(\lambda)$ is such that it leaves the linear system (3.1) invariant, i.e.,

$$
\partial_{x} \tilde{\hat{\psi}}=\tilde{\hat{U}}(x, t, \lambda) \tilde{\hat{\psi}}, \quad \partial_{t} \tilde{\hat{\psi}}=\tilde{\hat{V}}(x, t, \lambda) \tilde{\hat{\psi}}
$$

where $\widetilde{\hat{U}}$ and $\widetilde{\hat{V}}$ are given as

$$
\widetilde{\hat{U}}(x, t, \lambda)=\lambda \partial_{x} \widetilde{\hat{S}}, \quad \widetilde{\hat{V}}(x, t, \lambda)=[\widetilde{\hat{S}}, \tilde{\hat{G}}]+\lambda^{-1} \widetilde{\hat{G}}
$$

and $\tilde{S}$ and $\tilde{G}$ are the Lie algebra valued transformed matrix fields. By substituting equation (2.1) in equations (2.4), we get

$$
\widetilde{\hat{S}}=\hat{S}-\hat{M}, \quad \tilde{\hat{G}}=\hat{G} .
$$

As mentioned earlier equation (2.6) shows that $\hat{G}$ is a constant matrix and the matrix $M$ is subjected to satisfy the following equations

$$
\partial_{x} \hat{M} \hat{M}=\left[\partial_{x} \hat{S}, \hat{M}\right], \quad \partial_{t} \hat{M}=[[\hat{S}, \hat{G}], \hat{M}]+[\hat{G}, \hat{M}] \hat{M} .
$$


The matrix $M$ can be written in terms of the solutions of the linear system

$$
\hat{M}=\hat{\Theta} \Xi^{(-1) \dagger} \hat{\Theta}^{-1},
$$

and the Darboux transformation on the matrix fields $\hat{\psi}$ and $\hat{S}$ in hat space $\hat{\mathcal{V}}$ is

$$
\widetilde{\hat{\psi}}=\left(\lambda^{-1} I-\hat{\Theta} \Xi^{(-1) \dagger} \hat{\Theta}^{-1}\right) \hat{\psi}, \quad \tilde{\hat{S}}=\hat{S}-\hat{\Theta} \Xi^{(-1) \dagger} \hat{\Theta}^{-1} .
$$

From equation (3.8) we know that

$$
\hat{D}(\lambda) \hat{\psi}=D(\lambda) \psi
$$

which implies

$$
\hat{\psi}=\hat{D}^{-1}(\lambda) D(\lambda) \psi .
$$

The equation (3.9) relates the two solutions $\psi$ and $\hat{\psi}$. This transformation is known as the standard binary Darboux transformation and we write it as $B(\lambda)=\hat{D}^{-1}(\lambda) D(\lambda)$, i.e.

$$
\hat{\psi}=\hat{D}^{-1}(\lambda) D(\lambda) \psi=B(\lambda) \psi .
$$

By substituting (3.7), (2.2) in equation (3.10), we obtain the explicit transformation on $\psi$ as

$$
\begin{aligned}
\hat{\psi} & =\left(\lambda^{-1} I-\hat{\Theta} \Xi^{(-1) \dagger} \hat{\Theta}^{-1}\right)^{-1}\left(\lambda^{-1} I-\Theta \Lambda^{-1} \Theta^{-1}\right) \psi \\
& =\hat{\Theta}\left(\lambda^{-1} I-\Xi^{(-1) \dagger}\right)^{-1} \hat{\Theta}^{-1} \Theta\left(\lambda^{-1} I-\Lambda^{-1}\right) \Theta^{-1} \psi .
\end{aligned}
$$

By using (3.2) in equation (3.11), the expression of $\hat{\psi}$ may be simplified as

$$
\begin{aligned}
\hat{\psi} & =\Theta \Delta(\Theta, \Omega)^{-1}\left(\lambda^{-1} I-\Xi^{(-1) \dagger}\right)^{-1} \Delta(\Theta, \Omega) \Theta^{-1} \Theta\left(\lambda^{-1} I-\Lambda^{-1}\right) \Theta^{-1} \psi \\
& =\Theta \Delta(\Theta, \Omega)^{-1}\left(\lambda^{-1} I-\Xi^{(-1) \dagger}\right)^{-1} \Delta(\Theta, \Omega)\left(\lambda^{-1} I-\Lambda^{-1}\right) \Theta^{-1} \psi \\
& =\Theta \Delta(\Theta, \Omega)^{-1}\left(\lambda^{-1} I-\Xi^{(-1) \dagger}\right)^{-1}\left(\lambda^{-1} \Delta(\Theta, \Omega)-\Delta(\Theta, \Omega) \Lambda^{-1}\right) \Theta^{-1} \psi .
\end{aligned}
$$

By substituting the value of $\Delta(\Theta, \Omega) \Lambda^{-1}$ from (3.5), we get

$$
\begin{aligned}
\hat{\psi} & =\Theta \Delta(\Theta, \Omega)^{-1}\left(\lambda^{-1} I-\Xi^{(-1) \dagger}\right)^{-1}\left(\lambda^{-1} \Delta(\Theta, \Omega)-\Xi^{(-1) \dagger} \Delta(\Theta, \Omega)+\Omega^{\dagger} \Theta\right) \Theta^{-1} \psi \\
& =\left(I+\Theta \Delta(\Theta, \Omega)^{-1}\left(\lambda^{-1} I-\Xi^{(-1) \dagger}\right)^{-1} \Omega^{\dagger}\right) \psi=\left(I-\Theta \Delta(\Theta, \Omega)^{-1} \Delta(\cdot, \Omega)\right) \psi \\
& =\psi-\Theta \Delta(\Theta, \Omega)^{-1} \Delta(\psi, \Omega),
\end{aligned}
$$

where we have used equation (3.4) in obtaining the last step. Equation (3.12) may be written in terms of quasideterminant as

$$
\hat{\psi}=\left|\begin{array}{cc}
\Delta(\Theta, \Omega) & \Delta(\psi, \Omega) \\
\Theta & \psi
\end{array}\right| .
$$

The quasideterminant (3.13) is referred to as quasi-Grammian solution of the system. The adjoint binary transformation for $\hat{\phi} \in \hat{\mathcal{V}}^{\dagger}$ is obtained in a simmilar way and gives

$$
\hat{\phi}=\phi-\Omega \Delta(\Theta, \Omega)^{(-1) \dagger} \Delta^{\dagger}(\Theta, \phi)=\left|\begin{array}{cc}
\Delta^{\dagger}(\Theta, \Omega) & \Delta^{\dagger}(\Theta, \phi) \\
\Omega & \phi
\end{array}\right| .
$$

Again from equation (3.9), we have

$$
\hat{S}-\hat{\Theta} \Xi^{(-1) \dagger} \hat{\Theta}^{-1}=S-\Theta \Lambda^{-1} \Theta^{-1},
$$




$$
\hat{S}=S-\Theta \Lambda^{-1} \Theta^{-1}+\hat{\Theta} \Xi^{(-1) \dagger} \hat{\Theta}^{-1}=S-\Theta \Lambda^{-1} \Theta^{-1}+\Theta \Delta(\Theta, \Omega)^{-1} \Xi^{(-1) \dagger} \Delta(\Theta, \Omega) \Theta^{-1} .
$$

By using equation (3.5) for $\Xi^{(-1) \dagger} \Delta(\Theta, \Omega)$ in above equation

$$
\begin{aligned}
\hat{S} & =S-\Theta \Lambda^{-1} \Theta^{-1}+\Theta \Delta(\Theta, \Omega)^{-1}\left(\Delta(\Theta, \Omega) \Lambda^{-1}+\Omega^{\dagger} \Theta\right) \Theta^{-1} \\
& =S+\Theta \Delta(\Theta, \Omega)^{-1} \Omega^{\dagger}=S-\left|\begin{array}{cc}
\Delta(\Theta, \Omega) & \Omega^{\dagger} \\
\Theta & O
\end{array}\right| .
\end{aligned}
$$

For the next iteration of binary Darboux transformation, we take $\Theta_{1}, \Theta_{2}$ to be two particular solutions of the Lax pair (1.4) at $\Lambda=\Lambda_{1}$ and $\Lambda=\Lambda_{2}$ respectively. Similarly $\Omega_{1}, \Omega_{2}$ are two particular solutions of the Lax pair (2.10) at $\Xi=\Xi_{1}$ and $\Xi=\Xi_{2}$. Using the notation $\psi[1]=\psi$, $S[1]=S$ and $\psi[2]=\hat{\psi}, S[2]=\hat{S}$, we write two-fold binary Darboux transformation on $\psi$ as

$$
\psi[3]=\psi[2]-\Theta[2] \Delta(\Theta[2], \Omega[2])^{-1} \Delta(\psi[2], \Omega[2]),
$$

where $\Theta[1]=\Theta_{1}, \Omega[1]=\Omega_{1}, \Theta[2]=\left.\psi[2]\right|_{\psi \rightarrow \Theta_{2}}, \Omega[2]=\left.\phi[2]\right|_{\phi \rightarrow \Omega_{2}}$. Also note that by using the definition of the potential $\Delta$ and equation (3.6), we have

$$
\begin{aligned}
\Delta(\psi[2], \phi[2]) & =\Delta\left(\psi_{1}, \phi_{1}\right)-\Delta\left(\Theta_{1}, \phi_{1}\right) \Delta\left(\Theta_{1}, \Omega_{1}\right)^{-1} \Delta\left(\psi_{1}, \Omega_{1}\right) \\
& =\left|\begin{array}{cc}
\Delta\left(\Theta_{1}, \Omega_{1}\right) & \Delta\left(\psi, \Omega_{1}\right) \\
\Delta\left(\Theta_{1}, \phi\right) & \Delta(\psi, \phi)
\end{array}\right| .
\end{aligned}
$$

The equation (3.15) implies that

$$
\begin{aligned}
\Delta(\Theta[2], \Omega[2]) & =\Delta\left(\Theta_{2}, \Omega_{2}\right)-\Delta\left(\Theta_{1}, \Omega_{2}\right) \Delta\left(\Theta_{1}, \Omega_{1}\right)^{-1} \Delta\left(\Theta_{2}, \Omega_{1}\right) \\
& =\left|\begin{array}{cc}
\Delta\left(\Theta_{1}, \Omega_{1}\right) & \Delta\left(\Theta_{2}, \Omega_{1}\right) \\
\Delta\left(\Theta_{1}, \Omega_{2}\right) & \Delta\left(\Theta_{2}, \Omega_{2}\right)
\end{array}\right| .
\end{aligned}
$$

By using equations (3.15), (3.16) and the notation defined above in equation (3.14), we get

$$
\begin{aligned}
\psi[3]= & \left|\begin{array}{cc}
\Delta\left(\Theta_{1}, \Omega_{1}\right) & \Delta\left(\psi, \Omega_{1}\right) \\
\Theta_{1} & \psi
\end{array}\right|-\left|\begin{array}{cc}
\Delta\left(\Theta_{1}, \Omega_{1}\right) & \Delta\left(\Theta_{2}, \Omega_{1}\right) \\
\Theta_{1} & \Theta_{2}
\end{array}\right| \\
& \times\left|\begin{array}{ccc}
\Delta\left(\Theta_{1}, \Omega_{1}\right) & \Delta\left(\Theta_{2}, \Omega_{1}\right) \\
\Delta\left(\Theta_{1}, \Omega_{2}\right) & \Delta\left(\Theta_{2}, \Omega_{2}\right)
\end{array}\right| \begin{array}{cc}
\Delta\left(\Theta_{1}, \Omega_{1}\right) & \Delta\left(\psi, \Omega_{1}\right) \\
\Delta\left(\Theta_{1}, \phi\right) & \Delta\left(\psi, \Omega_{2}\right)
\end{array} \mid \\
= & \left|\begin{array}{ccc}
\Delta\left(\Theta_{1}, \Omega_{1}\right) & \Delta\left(\Theta_{2}, \Omega_{1}\right) & \Delta\left(\psi, \Omega_{1}\right) \\
\Delta\left(\Theta_{1}, \Omega_{2}\right) & \Delta\left(\Theta_{2}, \Omega_{2}\right) & \Delta\left(\psi, \Omega_{2}\right) \\
\Theta_{1} & \Theta_{2} & \psi
\end{array}\right|,
\end{aligned}
$$

where we have used the noncommutative Jacobi identity ${ }^{2}$ in obtaining (3.17). The $K$ th iteration of binary Darboux transformation leads to

$$
\begin{aligned}
\psi[K+1] & =\psi[K]-\Theta[K] \Delta(\Theta[K], \Omega[K])^{-1} \Delta(\psi[K], \Omega[K]) \\
& =\left|\begin{array}{cc}
\Delta(\Theta[K], \Omega[K]) & \Delta(\psi[K], \Omega[K]) \\
\Theta[K] & \psi[K]
\end{array}\right|
\end{aligned}
$$

${ }^{2}$ For quasideterminants, the noncommutative Jacobi identity is given as

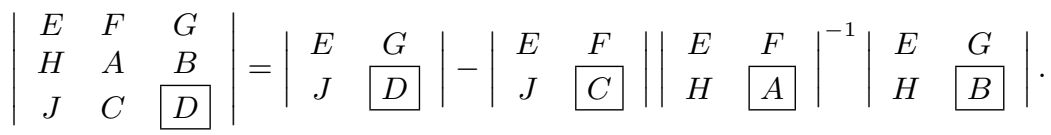

For the definition and more properties of quasideterminants see e.g. [9, 10, 11, 12, 28]. 


$$
=\left|\begin{array}{cccc}
\Delta\left(\Theta_{1}, \Omega_{1}\right) & \cdots & \Delta\left(\Theta_{K}, \Omega_{1}\right) & \Delta\left(\psi, \Omega_{1}\right) \\
\vdots & \cdots & \vdots & \vdots \\
\Delta\left(\Theta_{1}, \Omega_{K}\right) & \cdots & \Delta\left(\Theta_{K}, \Omega_{K}\right) & \Delta\left(\psi, \Omega_{K}\right) \\
\Theta_{1} & \cdots & \Theta_{K} & \psi
\end{array}\right| .
$$

Above result can be proved by induction by using the properties of quasideterminants. Similarly the $K$ th iteration of adjoint binary Darboux transformation gives

$$
\begin{aligned}
\phi[K+1] & =\phi[K]-\Omega[K] \Delta(\Theta[K], \Omega[K])^{(-1) \dagger} \Delta(\Theta[K], \phi[K])^{\dagger} \\
= & \left|\begin{array}{ccccc}
\Delta(\Theta[K], \Omega[K])^{\dagger} & \Delta(\Theta[K], \phi[K])^{\dagger} \\
\Omega[K] & {[\phi[K]}
\end{array}\right| \\
= & \left|\begin{array}{ccccc}
\Delta\left(\Theta_{1}, \Omega_{1}\right)^{\dagger} & \Delta\left(\Theta_{2}, \Omega_{1}\right)^{\dagger} & \cdots & \Delta\left(\Theta_{K}, \Omega_{1}\right)^{\dagger} & \Delta\left(\Theta_{1}, \phi\right)^{\dagger} \\
\Delta\left(\Theta_{1}, \Omega_{2}\right)^{\dagger} & \Delta\left(\Theta_{2}, \Omega_{2}\right)^{\dagger} & \cdots & \Delta\left(\Theta_{K}, \Omega_{2}\right)^{\dagger} & \Delta\left(\Theta_{2}, \phi\right)^{\dagger} \\
\vdots & \vdots & \cdots & \vdots & \vdots \\
\Delta\left(\Theta_{1}, \Omega_{K}\right)^{\dagger} & \Delta\left(\Theta_{2}, \Omega_{K}\right)^{\dagger} & \cdots & \Delta\left(\Theta_{K}, \Omega_{K}\right)^{\dagger} & \Delta\left(\Theta_{K}, \phi\right)^{\dagger} \\
\Omega_{1} & \Omega_{2} & \cdots & \Omega_{K} & \phi
\end{array}\right| .
\end{aligned}
$$

The multisoliton $S[K+1]$ can be obtained by putting $\lambda=0$ in the expression for $\psi[K+1](3.18)$ and using $G=\left.\psi\right|_{\lambda=0}$, which on silmplification gives

$$
S[K+1]=S-\left|\begin{array}{cccc}
\Delta\left(\Theta_{1}, \Omega_{1}\right) & \cdots & \Delta\left(\Theta_{K}, \Omega_{1}\right) & \Omega_{1}^{\dagger} \\
\vdots & \cdots & \vdots & \vdots \\
\Delta\left(\Theta_{1}, \Omega_{K}\right) & \cdots & \Delta\left(\Theta_{K}, \Omega_{K}\right) & \Omega_{K}^{\dagger} \\
\Theta_{1} & \cdots & \Theta_{K} & I
\end{array}\right| .
$$

Similar expression can be obtained for the $K$ th iteration of $S^{\dagger}$.

Therefore by using the standard binary Darboux transformation we have obtained the grammian type solutions for the linear system and the potential is also expressed in terms of quasideterminants. That is by constructing binary Darboux transformation in terms of spectral parameter we can get the expression of the matrix solution of the linear system in terms of grammian type quasideterminants which is different in representation from the solutions obtained by elementary Darboux transformation. In addition to the solutions of the linear system we are also able to obtain explicit quasideterminant expression of the potential $\Delta$ in terms of the particular solutions of the linear system. It is important to note that the spectral parameter remains unchanged in binary Darboux transformation. We consider the eigenfunctions (solutions of direct Lax pair) and adjoint eigenfunctions (solutions of adjoint pair). The bilinear potential $\Delta$ is related to each pair of (direct and adjoint) solutions. Since we know that the solutions of the linear system can be column vectors or they can be combined to give solution in matrix form. As in the present case when the solutions are in matrix form the potential $\Delta$ is also a matrix. It has been shown earlier that matrix solutions can be reduced to vector solutions [15]. In such a case when solutions are vectors the potential $\Delta$ becomes scalar and by replacing spectral parameter with derivative we can consider potential to be a contour integration of the corresponding expressions in $x-t$ plane. In the next section we will see what happens when we apply our method to a specific case of $S U(2)$ system.

\section{Explicit solutions for the $S U(2)$ system}

In this section we consider the generalized coupled dispersionless integrable system based on the Lie group $S U(2)$ and calculate the soliton solutions by using binary Darboux transformation. 
For the Lie group $S U(2)$ the matrix fields $S$ and $G$ are valued in the Lie algebra $\mathbf{s u}(2)$ and we have

$$
\begin{array}{cc}
S^{\dagger}=-S, & G^{\dagger}=-G, \\
\operatorname{Tr} S=0, & \operatorname{Tr} G=0 .
\end{array}
$$

Following the same steps for the direct Lax pair as obtained in [16]. We define a vector $\phi=$ $\left(\phi_{1}, \phi_{2}, \phi_{3}\right)$ in such a way that the matrix field $S$ is given by

$$
S=i\left(\begin{array}{cc}
\phi_{3} & \phi_{1}-i \phi_{2} \\
\phi_{1}+i \phi_{2} & -\phi_{3}
\end{array}\right)
$$

Equation (4.3) satisfies the conditions (4.1) and (4.2). The matrices $U$ and $V$ are then given as

$$
U=i \lambda\left(\begin{array}{cc}
\partial_{x} \phi_{3} & \partial_{x} \phi_{1}-i \partial_{x} \phi_{2} \\
\partial_{x} \phi_{1}+i \partial_{x} \phi_{2} & -\partial_{x} \phi_{3}
\end{array}\right), \quad V=\left(\begin{array}{cc}
0 & \phi_{1}-i \phi_{2} \\
-\phi_{1}-i \phi_{2} & 0
\end{array}\right)-\frac{i}{2 \lambda}\left(\begin{array}{cc}
1 & 0 \\
0 & -1
\end{array}\right) .
$$

By writing $\phi_{1}=r, \phi_{2}=0$ and $\phi_{3}=q$, we get the coupled dispersionless integrable system as given in [24]

$$
\partial_{x} \partial_{t} q+2 \partial_{x} r r=0, \quad \partial_{x} \partial_{t} r-2 \partial_{x} q r=0
$$

and the matrix $S$ from equation (4.3) is given as

$$
S=i\left(\begin{array}{cc}
q & r \\
r & -q
\end{array}\right)
$$

To obtain the expression for the Darboux matrix we take

$$
\Lambda=\left(\begin{array}{cc}
\lambda_{1} & 0 \\
0 & -\lambda_{1}
\end{array}\right), \quad \Theta=\left(\begin{array}{cc}
\alpha & \beta \\
\beta & -\alpha
\end{array}\right) .
$$

By using equations (4.5) and (2.7) in equation (2.2), we get

$$
D(\lambda)=\left(\begin{array}{cc}
\lambda^{-1}-\lambda_{1}^{-1} \cos \omega & -\lambda_{1}^{-1} \sin \omega \\
-\lambda_{1}^{-1} \sin \omega & \lambda^{-1}-\lambda_{1}^{-1} \cos \omega
\end{array}\right)
$$

where we have assumed that $\tan \frac{\omega}{2}=\frac{\beta}{\alpha}$. We now consider the seed solution as follows

$$
\psi=\left(\begin{array}{cc}
e^{i \lambda x-\frac{i}{2 \lambda} t} & 0 \\
0 & e^{-i \lambda x+\frac{i}{2 \lambda} t}
\end{array}\right) .
$$

By using the above equation (4.6) we can write the particular matrix solution $\Theta$ of the direct Lax pair (1.4) as

$$
\Theta=\left(\psi\left(\lambda_{1}\right)|1\rangle \quad \psi\left(\lambda_{2}\right)|2\rangle\right)=\left(\begin{array}{cc}
e^{i \lambda_{1} x-\frac{i}{2 \lambda_{1}} t} & e^{i \lambda_{2} x-\frac{i}{2 \lambda_{2}} t} \\
e^{-i \lambda_{1} x+\frac{i}{2 \lambda_{1}} t} & -e^{-i \lambda_{2} x+\frac{i}{2 \lambda_{2}} t}
\end{array}\right) .
$$

By substituting $\lambda_{2}=-\lambda_{1}$ we get from above equation (4.7)

$$
\Theta=\left(\begin{array}{cc}
e^{i \lambda_{1} x-\frac{i}{2 \lambda_{1}} t} & e^{-i \lambda_{1} x+\frac{i}{2 \lambda_{1}} t} \\
e^{-i \lambda_{1} x+\frac{i}{2 \lambda_{1}} t} & -e^{i \lambda_{1}-\frac{i}{2 \lambda_{1}} t}
\end{array}\right)
$$


Taking $l=2 i \lambda_{1} x-\frac{i}{\lambda_{1}} t$ and using the definition (2.7) we get from (4.8)

$$
M=\frac{\lambda_{1}^{-1}}{2 \cosh l}\left(\begin{array}{cc}
2 \sinh l & 2 \\
2 & -2 \sinh l
\end{array}\right)=\lambda_{1}^{-1}\left(\begin{array}{cc}
\tanh l & \operatorname{sech} l \\
\operatorname{sech} l & -\tanh l
\end{array}\right) .
$$

From equation (2.5) we have

$$
\partial_{x} S[1]=\partial_{x} S-\partial_{x} M
$$

On comparison with equation (4.4) and using (4.9) we obtain

$$
\begin{aligned}
\partial_{x} q[1] & =\partial_{x} q+i \partial_{x} M_{11}=1+i \lambda_{1}^{-1} \partial_{x} \tanh l=1-2 \operatorname{sech}^{2} l \\
& =1-2 \operatorname{sech}^{2}\left(2 i \lambda_{1} x-\frac{i}{\lambda_{1}} t\right)
\end{aligned}
$$

where we have used $\partial_{x} q=1$. Similarly we have for $r=0$

$$
\partial_{x} r[1]=\partial_{x} r+i \partial_{x} M_{12}
$$

which gives

$$
r[1]=i M_{12}=i \lambda_{1}^{-1} \operatorname{sech}\left(2 i \lambda_{1} x-\frac{i}{\lambda_{1}} t\right) .
$$

It is easy to show from equations (4.10) and (4.11) that in the asymptotic limit $\partial_{x} q[1] \rightarrow 1$ and $r[1] \rightarrow 0$. Now by making use of above calculation we can write the iterated solution $\psi[1]$ as

$$
\psi[1]=D(\lambda) \psi=\left(\begin{array}{cc}
\left(\lambda^{-1}-\lambda_{1}^{-1} \tanh l\right) e^{\frac{l}{2}} & -\lambda_{1}^{-1} \operatorname{sech} l e^{\frac{-l}{2}} \\
-\lambda_{1}^{-1} \operatorname{sech} e^{\frac{l}{2}} & \left(\lambda^{-1}+\lambda_{1}^{-1} \tanh l\right) e^{\frac{-l}{2}}
\end{array}\right) .
$$

Repeating the calculations as we did for direct pair, we get

$$
\Omega=\left(\begin{array}{cc}
e^{\frac{p}{2}} & e^{-\frac{p}{2}} \\
e^{-\frac{p}{2}} & -e^{\frac{p}{2}}
\end{array}\right)
$$

where $p=2 i \xi_{1} x-\frac{i}{\xi_{1}} t$. To obtain the expression for $\hat{S}$, we start with the definition (3.6) of $\Delta(\Theta, \Omega), \bar{\xi}=-\xi$ and by using (4.8), (4.12) obtain for the present case

$$
\Delta(\Theta, \Omega)=\left(\begin{array}{cc}
-\frac{2 \cosh \hat{l}}{\xi_{1}^{-1}+\lambda_{1}^{-1}} & -\frac{2 \sinh \hat{p}}{\xi_{1}^{-1}-\lambda_{1}^{-1}} \\
-\frac{2 \sinh \hat{p}}{\xi_{1}^{-1}-\lambda_{1}^{-1}} & \frac{2 \cosh \hat{l}}{\xi_{1}^{-1}+\lambda_{1}^{-1}}
\end{array}\right)
$$

where

$$
\hat{l}\left(x^{+}, x^{-}\right)=i\left(\xi_{1}+\lambda_{1}\right) x-\frac{i}{2}\left(\frac{1}{\xi_{1}}+\frac{1}{\lambda_{1}}\right) t, \quad \hat{p}\left(x^{+}, x^{-}\right)=i\left(\xi_{1}-\lambda_{1}\right) x-\frac{i}{2}\left(\frac{1}{\xi_{1}}-\frac{1}{\lambda_{1}}\right) t .
$$

Now we consider

$$
\hat{M}=\Theta \Delta(\Theta, \Omega)^{-1} \Omega^{\dagger}=\left(\begin{array}{ll}
\hat{M}_{11} & \hat{M}_{12} \\
\hat{M}_{21} & \hat{M}_{22}
\end{array}\right)
$$




$$
=\frac{4}{K}\left(\begin{array}{cc}
\frac{\cosh \hat{l} \sinh \hat{l}}{\xi_{1}^{-1}+\lambda_{1}^{-1}}+\frac{\cosh \hat{p} \sinh \hat{p}}{\xi_{1}^{-1}-\lambda_{1}^{-1}} & \frac{\cosh \hat{l} \cosh \hat{p}}{\xi_{1}^{-1}+\lambda_{1}^{-1}}-\frac{\sinh \hat{p} \sinh \hat{l}}{\xi_{1}^{-1}-\lambda_{1}^{-1}} \\
\frac{\cosh \hat{l} \cosh \hat{p}}{\xi_{1}^{-1}+\lambda_{1}^{-1}}-\frac{\sinh \hat{p} \sinh \hat{l}}{\xi_{1}^{-1}-\lambda_{1}^{-1}} & -\frac{\cosh \hat{l} \sinh \hat{l}}{\xi_{1}^{-1}+\lambda_{1}^{-1}}-\frac{\cosh \hat{p} \sinh \hat{p}}{\xi_{1}^{-1}-\lambda_{1}^{-1}}
\end{array}\right)
$$

where

$$
\begin{aligned}
& K=\operatorname{det} \Delta(\Theta, \Omega)=\frac{-4 \cosh ^{2} \hat{l}}{\left(\xi_{1}^{-1}+\lambda_{1}^{-1}\right)^{2}}-\frac{4 \sinh ^{2} \hat{p}}{\left(\xi_{1}^{-1}-\lambda_{1}^{-1}\right)^{2}}, \quad \hat{S}=S+\Theta \Delta(\Theta, \Omega)^{-1} \Omega^{\dagger}, \\
& \left(\begin{array}{cc}
i q[1] & i r[1] \\
i r[1] & -i q[1]
\end{array}\right)=\left(\begin{array}{cc}
i q+\hat{M}_{11} & i r+\hat{M}_{12} \\
i r+\hat{M}_{21} & -i q+\hat{M}_{22}
\end{array}\right)
\end{aligned}
$$

From equation (4.14) and (4.13) by using $\partial_{x} q=1$ and $r=0$ we get

$$
\begin{aligned}
& \partial_{x} q[1]=1+\frac{8 \lambda \xi}{K}\left[\sinh p \sinh l+\frac{2}{K}\left\{\frac{\sinh 2 \hat{l}}{\xi_{1}^{-1}+\lambda_{1}^{-1}}-\frac{\sinh 2 \hat{p}}{\xi_{1}^{-1}-\lambda_{1}^{-1}}\right\}\right], \\
& r[1]=-i \frac{4}{K}\left\{\frac{\cosh \hat{l} \cosh \hat{p}}{\xi_{1}^{-1}+\lambda_{1}^{-1}}-\frac{\sinh \hat{p} \sinh \hat{l}}{\xi_{1}^{-1}-\lambda_{1}^{-1}}\right\} .
\end{aligned}
$$

In the asymptotic limit for $t \rightarrow \pm \infty$, we have $\hat{l} \rightarrow \pm \infty$ and the equations (4.15) and (4.16) become

$$
\lim _{\hat{l} \rightarrow \pm \infty} \partial_{x} q[1]=1, \quad \lim _{\hat{l} \rightarrow \pm \infty} r[1]=0 .
$$

We see that in the asymptotic limit, we get much simpler expressions. Note that the expression is similar to the one we obtain from elementary Darboux transformation. Now we consider the special case when $\xi=\lambda$ which gives $\hat{p}=0$. The solutions (4.15) and (4.16) become

$$
\begin{aligned}
& \partial_{x} q[1]=1+2 \operatorname{sech}^{2} \hat{l}, \\
& r[1]=i \lambda^{-1} \operatorname{sech} \hat{l} .
\end{aligned}
$$

On comparison of equations (4.10) and (4.11) with equations (4.15) and (4.16) we see that the original solutions obtained by the standard binary Darboux transformation are different from those of elementary Darbouix transformation and contain the contribution from both the direct and adjoint system. If we take $\xi=\lambda$ the solutions from both the techniques become equal as shown by equations (4.18) and (4.19). Therefore the advantage of using standard binary Darboux transformation is that we can obtain the solutions in the form of direct and adjoint space parameters and then without using elementary Darboux transformation we can obtain solutions just by equating parameters as shown above where we have obtained equations (4.18) and (4.19) (which have same form as solutions obtained from elementary Darboux transformation) from equations (4.15) and (4.16) (which give solutions by standard binary Darboux transformation).

It is simple to show that the solutions (4.18) and (4.19) have the same behaviour in asymptotic limit and satisfy (4.17). Plots of solutions (4.18) and (4.19) for $\lambda=i$ are shown in Figs. 1 and 2. Now we show the relationship between our solution of the system (1.3) and the solution $\phi$ of the sine-Gordon equation. The sine-Gordon equation is given as

$$
\partial_{x} \partial_{t} \phi=2 \sin \phi
$$

and is related to our system by the following equations

$$
\partial_{x} q=\cos \phi, \quad r= \pm \frac{1}{2} \partial_{t} \phi
$$




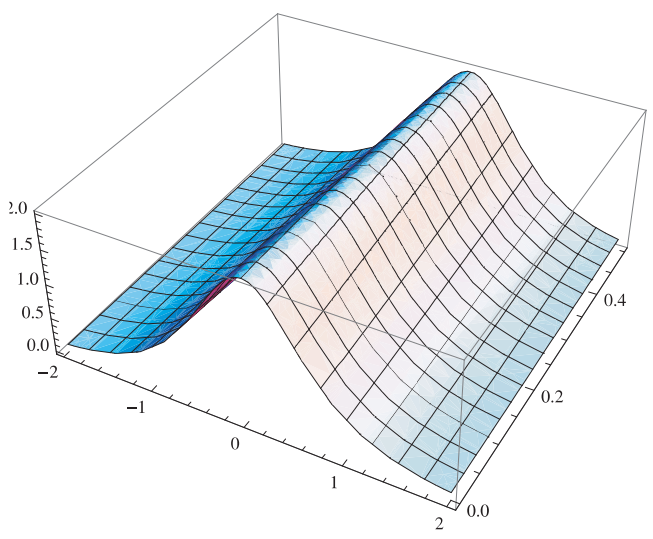

Figure 1. Plot of solution (4.18) representing one soliton solution $\partial_{x} q[1]$.

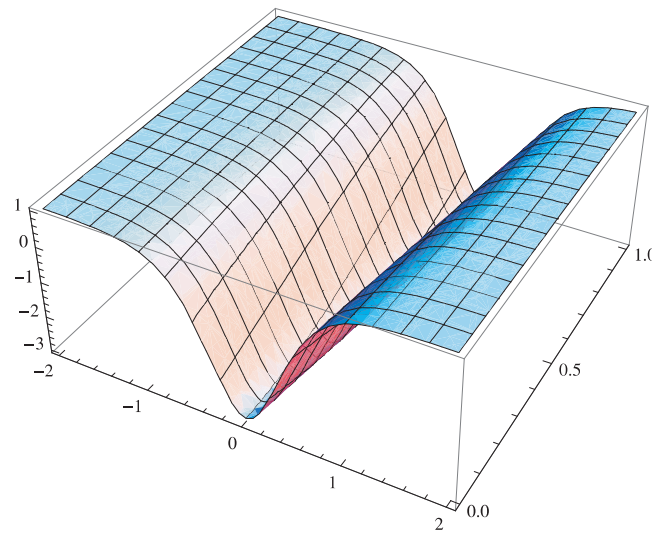

Figure 2. Plot of solution (4.19) representing one soliton solution $r[1]$.

To obtain the expression for $\phi[1]$, we use equation (4.20) in equation (4.19) which gives

$$
\begin{aligned}
& \pm \frac{1}{2} \partial_{t} \phi[1]=i \lambda^{-1} \operatorname{sech} \hat{l}, \\
& \phi[1]= \pm 2 i \lambda^{-1} \int \operatorname{sech}\left(2 i \lambda x-\frac{i}{\lambda} t\right) d t= \pm \frac{2}{\lambda^{2}} \tan ^{-1}\left(\exp \left(2 i \lambda x-\frac{i}{\lambda} t\right)\right) .
\end{aligned}
$$

The equation (4.21) is the one-kink solution to the sine-Gordon equation [33].

\section{Conclusions}

In this paper, we have composed the elementary Darboux transformations of the generalized coupled dispersionless system and obtained the standard binary Darboux transformation of the model. By iterating the standard binary Darboux transformation we have generated the multisolitons of the model. We have also obtained the quasideterminant expression for the potential $\Delta$. We have also considered the case of coupled dispersionless integrable system based on the Lie group $S U(2)$, and have obtained explicit expressions of Grammian solutions of the system. There are various directions in which the the integrability properties of the generalized dispersionless integrable system can be studied. One such study is to investigate the r-matrix structure and the existence of infinitely many conservation laws of the system. We shall return these and related investigations in a separate work.

\section{Acknowledgements}

BH would like to thank Department of Physics, University of the Punjab, Lahore, Pakistan for providing the research facilities.

\section{References}

[1] Aoyama S., Kodama Y., Topological conformal field theory with a rational $W$ potential and the dispersionless KP hierarchy, Modern Phys. Lett. A 9 (1994), 2481-2492, hep-th/9404011.

[2] Carroll R., Kodama Y., Solution of the dispersionless Hirota equations, J. Phys. A: Math. Gen. 28 (1995), 6373-6387, hep-th/9506007.

[3] Cieśliński J., An algebraic method to construct the Darboux matrix, J. Math. Phys. 36 (1995), 5670-5706.

[4] Cieśliński J., Biernacki W., A new approach to the Darboux-Bäcklund transformation versus the standard dressing method, J. Phys. A: Math. Gen. 38 (2005), 9491-9501, nlin.SI/0506016. 
[5] Darboux G., Leçons sur la théorie générale des surfaces et les applications géométriques du calcul infinitésimal. Vol. IV. Deformation infiniment petite et représentation sphérique, Gauthier-Villars, Paris, 1896.

[6] Darboux G., Sur une proposition relative aux équations linéaires, C. R. Acad. Sci. Paris 94 (1882), 14561459.

[7] Doktorov E.V., Leble S.B., A dressing method in mathematical physics, Mathematical Physics Studies, Vol. 28, Springer, Dordrecht, 2007.

[8] Dunajski M., An interpolating dispersionless integrable system, J. Phys. A: Math. Theor. 41 (2008), 315202, 9 pages, arXiv:0804.1234.

[9] Gel'fand I., Gel'fand S., Retakh V., Wilson R.L., Quasideterminants, Adv. Math. 193 (2005), 56-141, math.QA/0208146.

[10] Gel'fand I., Retakh V., Determinants of matrices over noncommutative rings, Funct. Anal. Appl. 25 (1991), 91-102.

[11] Gel'fand I., Retakh V., Theory of noncommutative determinants, and characteristic functions of graphs, Funct. Anal. Appl. 26 (1992), 231-246.

[12] Gel'fand I., Retakh V., Wilson R.L., Quaternionic quasideterminants and determinants, in Lie Groups and Symmetric Spaces, Amer. Math. Soc. Transl. Ser. 2, Vol. 210, Amer. Math. Soc., Providence, RI, 2003, 111-123, math.QA/0206211.

[13] Haider B., Hassan M., Quasideterminant solutions of an integrable chiral model in two dimensions, J. Phys. A: Math. Theor. 42 (2009), 355211, 18 pages, arXiv:0912.3071.

[14] Haider B., Hassan M., The U(N) chiral model and exact multi-solitons, J. Phys. A: Math. Theor. 41 (2008), 255202, 17 pages, arXiv:0912.1984.

[15] Haider B., Hassan M., Saleem U., Binary Darboux transformation and quasideterminant solutions of the chiral field, J. Nonlinear Math. Phys., to appear.

[16] Hassan M., Darboux transformation of the generalized coupled dispersionless integrable system, J. Phys. A: Math. Theor. 42 (2009), 065203, 11 pages, arXiv:0912.1671.

[17] Hirota R., Tsujimoto S., Note on "New coupled integrable dispersionless equations", J. Phys. Soc. Japan 63 (1994), 3533.

[18] Ji Q., Darboux transformation for MZM-I, II equations, Phys. Lett. A 311 (2003), 384-388.

[19] Kakuhata H., Konno K., A generalization of coupled integrable, dispersionless system, J. Phys. Soc. Japan 65 (1996), 340-341.

[20] Kakuhata H., Konno K., Canonical formulation of a generalized coupled dispersionless system, J. Phys. A: Math. Gen. 30 (1997), L401-L407.

[21] Kakuhata H., Konno K., Lagrangian, Hamiltonian and conserved quantities for coupled integrable, dispersionless equations, J. Phys. Soc. Japan 65 (1996), 1-2.

[22] Kodama Y., A method for solving the dispersionless KP equation and its exact solutions, Phys. Lett. A 129 (1988), 223-226.

[23] Kodama Y., Pierce V.U., Combinatorics of dispersionless integrable systems and universality in random matrix theory, Comm. Math. Phys. 292 (2009), 529-568, arXiv:0811.0351.

[24] Konno K., Oono H., New coupled integrable dispersionless equations, J. Phys. Soc. Japan 63 (1994), 377378.

[25] Konopelchenko B.G., Magri F., Coisotropic deformations of associative algebras and dispersionless integrable hierarchies, Comm. Math. Phys. 274 (2007), 627-658, nlin.SI/0606069.

[26] Kotlyarov V.P., On equations gauge equivalent to the sine-Gordon and Pohlmeyer-Lund-Regge equations, J. Phys. Soc. Japan 63 (1994), 3535-3537.

[27] Krichever I.M., The dispersionless Lax equations and topological minimal models, Comm. Math. Phys. 143 (1992), 415-429.

[28] Krob D., Leclerc B., Minor identities for quasi-determinants and quantum determinants, Comm. Math. Phys. 169 (1995), 1-23, hep-th/9411194.

[29] Leble S.B., Binary Darboux transformations and systems of $N$ waves in rings, Theoret. and Math. Phys. 122 (2000), 200-210. 
[30] Leble S.B., Elementary and binary Darboux transformations at rings, Comput. Math. Appl. 35 (1998), 73-81.

[31] Leble S.B., Ustinov N.V., Darboux transforms, deep reductions and solitons, J. Phys. A: Math. Gen. 26 (1993), 5007-5016.

[32] Leble S.B., Ustinov N.V., Deep reductions for matrix Lax system, invariant forms and elementary Darboux transforms, in Proceedings of NEEDS-92 Workshop, World Scientific, Singapore, 1993, 34-41.

[33] Li C.X., Nimmo J.J.C., Quasideterminant solutions of a non-abelian Toda lattice and kink solutions of a matrix sine-Gordon equation, Proc. R. Soc. Lond. Ser. A 464 (2008), 951-966, arXiv:0711.2594.

[34] Mañas M., Darboux transformations for the nonlinear Schrödinger equations, J. Phys. A: Math. Gen. 29 (1996), 7721-7737.

[35] Matveev V.B., Darboux invariance and the solutions of Zakharov-Shabat equations, Preprint LPTHE 79-07, LPTHE, Orsay, 1979, 11 pages.

[36] Matveev V.B., Darboux transformation and explicit solutions of the Kadomtcev-Petviaschvily equation, depending on functional parameters, Lett. Math. Phys. 3 (1979), 213-216.

[37] Matveev V.B., Darboux transformation and the explicit solutions of differential-difference and differencedifference evolution equations. I, Lett. Math. Phys. 3 (1979), 217-222.

[38] Matveev V.B., Salle M.A., Differential-difference evolution equations. II. Darboux transformation for the Toda lattice, Lett. Math. Phys. 3 (1979), 425-429.

[39] Matveev V.B., Salle M.A., Darboux transformations and solitons, Springer Series in Nonlinear Dynamics, Springer-Verlag, Berlin, 1991.

[40] Nimmo J.J.C., Darboux transformations for discrete systems, Chaos Solitons Fractals 11 (2000), 115-120.

[41] Nimmo J.J.C., Darboux transformations from reductions of the KP hierarchy, in Nonlinear Evolution Equations \& Dynamical Systems: NEEDS '94 (Los Alamos, NM), World Sci. Publ., River Edge, NJ, 1995, 168-177, solv-int/9410001.

[42] Nimmo J.J.C., Gilson C.R., Ohta Y., Applications of Darboux transformations to the self-dual Yang-Mills equations, Theoret. and Math. Phys. 122 (2000), 239-246.

[43] Oevel W., Schief W., Darboux theorems and the KP hierarchy, in Applications of Analytic and Geometric Methods to Nonlinear Differential Equations (Exeter, 1992), NATO Adv. Sci. Inst. Ser. C Math. Phys. Sci., Vol. 413, Kluwer Acad. Publ., Dordrecht, 1993, 193-206.

[44] Park Q.H., Shin H.J., Darboux transformation and Crum's formula for multi-component integrable equations, Phys. D 157 (2001), 1-15.

[45] Rogers C., Schief W.K., Bäcklund and Darboux transformations: geometry and modern applications in soliton theory, Cambridge Texts in Applied Mathematics, Cambridge University Press, Cambridge, 2002.

[46] Sakhnovich A.L., Dressing procedure for solutions of nonlinear equations and the method of operator identities, Inverse Problems 10 (1994), 699-710.

[47] Takasaki K., Dispersionless Toda hierarchy and two-dimensional string theory, Comm. Math. Phys. 170 (1995), 101-116, hep-th/9403190.

[48] Takasaki K., Takebe T., Integrable hierarchies and dispersionless limit, Rev. Math. Phys. 7 (1995), 743-808, hep-th/9405096.

[49] Ustinov N.V., The reduced self-dual Yang-Mills equation, binary and infinitesimal Darboux transformations, J. Math. Phys. 39 (1998), 976-985.

[50] Wiegmann P.B., Zabrodin A., Conformal maps and integrable hierarchies, Comm. Math. Phys. 213 (2000), 523-538, hep-th/9909147. 